\title{
O PACIENTE RENAL CRÔNICO E SUA RELAÇÃO COM O TRABALHO'
}

[The chronic renal patient and the development of the work]

\author{
Eli Alves de Lara* \\ Leila Maria Mansano Sarquis**
}

RESUMO: O presente estudo buscou identificar as mudanças que a hemodiálise acarretou para a realização de atividades laborais por pacientes renais crônicos de um Centro de Hemodiálise em Curitiba. O estudo foi descritivo quantitativo. A população estudada foi de 43 pacientes que faziam sessões de hemodiálise. Nos resultados obtidos foram evidenciadas as dificuldades que estes pacientes apresentam para manter a atividade laboral após iniciar o tratamento. A maior parte da população estudada está aposentada por invalidez, recebe auxílio-doença ou aguarda aposentadoria. Os pacientes entrevistados que possuem atividade laboral referem ter dificuldades para realizá-las devido à impossibilidade de manter uma jornada de trabalho adequada. Também apresentam fraqueza após as sessões de hemodiálise. Ficou caracterizado que a maioria dos pacientes tem dificuldades para se manter financeiramente, uma vez que não conseguem desenvolver as atividades laborais.

PALAVRAS-CHAVE: Hemodiálise; Insuficiência renal crônica; Saúde do trabalhador.

\section{INTRODUÇÃO}

O trabalho ou atividade laboral, segundo Rodrigues (1995, p.11) "ocupa um espaço muito

\footnotetext{
${ }^{1}$ Estudo realizado a partir da Monografia de Conclusão do Curso de Enfermagem da Universidade Federal do Paraná.

*Acadêmica de Enfermagem da Universidade Federal do Paraná.

**Enfermeira. Profa. do Departamento de Enfermagem da Universidade Federal do Paraná, membro do GEMSA (Grupo Estudo Multidisciplinar da Saúde do Adulto), doutoranda do Programa de Pós-graduação na área de Concentração em Administração de Enfermagem pela EEUSP, bolsista do CNPq.
}

importante na vida de todos nós, ou seja, quase todo mundo trabalha, e uma grande parte de nossa vida é passada dentro do local de trabalho".O trabalho ou atividade laboral não tem somente um papel na subsistência dos indivíduos, mas também é percebido como algo que dá sentido à vida e impulsiona o crescimento humano.

Para o paciente renal crônico desempenhar esta tarefa tão comum e cotidiana à maioria da população às vezes se torna muito complicado, devido ao tempo que essas pessoas têm que passar conectadas à máquina de hemodiálise e devido aos possíveis problemas que o tratamento ocasiona. Estes indivíduos não podem cumprir a jornada de trabalho exigida ou muitas vezes não podem realizar as atividades que o ambiente de trabalho requer, por comprometimento físico e também por alterações fisiológicas decorrentes dos problemas pertencentes aos renais crônicos.

$O$ interesse pelo tema surgiu da necessidade de conhecer uma parte da vida destes pacientes que compromete a subsistência e a identidade pessoal e qual a situação em que eles estão perante o vinculo empregatício, pois muitos não possuem emprego, adquiriram aposentadoria por invalidez e outros fatores ainda que comprometeram suas atividades laborais.

O objetivo deste estudo foi identificar as principais mudanças que a hemodiálise acarretou para a vida do renal crônico e caracterizar a importância do trabalho para o homem nos aspectos físicos, mentais e sociais.

\section{REVISÃO DE LITERATURA}

Os rins são essenciais à homeostase, não 
apenas porque eliminam produtos indesejáveis do metabolismo, como também por manter constantes o volume extracelular, a concentração extracelular de potássio, a pressão osmótica e o equilíbrio ácido-base do organismo, além de desempenhar um papel fundamental na regulação da pressão arterial e de desempenhar funções endócrinas como a produção de eritropoetina e da forma ativa da vitamina $D$.

Para Zatz (2002, p.261) o individuo em insuficiência renal retém progressivamente os produtos do metabolismo nitrogenado como uréia, acumula líquidos sob a forma de edema, perde a capacidade de diluir e concentrar a urina e tornase incapaz de regular o equilíbrio ácido-base ou de manter o balanço de níveis plasmáticos de eletrólitos tais como sódio, potássio, magnésio, cálcio e fósforo. Ainda segundo o autor, “... se a perda de função renal ocorrer lentamente devido á perda progressiva de nefróns (neuropatias progressivas), os nefróns remanescentes podem adaptar-se e manter a homeostase durante um longo período, desenvolvendo uma insuficiência crônica".

Segundo Guyton \& Hall (1996, p.379), as principais causas da IRC são a glomerulonefrite (distúrbio imunológico), diabetes melitus e a hipertensão arterial, sendo as duas últimas as principais causas do estágio terminal da doença. O tratamento utilizado para a Insuficiência Renal Crônica é a diálise, que consiste na depuração sanguínea através de membranas semipermeáveis naturais (peritônio) ou extracorpóreas (filtros de hemodiálise/ hemofiltração), aplicada em substituição á função renal. A diálise permite a remoção de substâncias tóxicas e de fluidos (ultrafiltrados), a fim de manter o equilíbrio ácido-base, eletrolítico e volêmico. A remoção das toxinas e fluidos se faz por dois princípios fisiológicos: a difusão e a ultrafiltração. A hemodiálise segundo Fermi (2003, p-37), “...é feita entre membranas derivadas de celulose que atuam como membrana semipermeável. Essa membrana encontra-se imersa em uma solução eletrolítica (solução de diálise ou banhos de diálise) que possui concentração semelhante ao plasma de um individuo com função renal normal." Para realizar a hemodiálise são necessários: rim artificial (máquina), água tratada, solução de diálise, dialisador (capilar) e via de acesso.

Segundo Riella (1996, p. 617), “...a substituição da função renal por uma máquina é imperfeita e sujeita a problemas agudos, decorrentes do uso de circuito extracorpóreo, e também ao aparecimento de sintoma e sinais em longo prazo, por alterações metabólicas não corrigidas ou surgidas no processo." Existem complicações agudas e crônicas do procedimento hemodialitico. As complicações agudas mais comuns são: instabilidade vascular, hipotensão arterial, cefaléia intensa, náuseas e câimbras, todas associadas à retirada de solutos e água do organismo. As complicações crônicas mais comuns são decorrentes da própria IRC ou por exposição a produtos químicos ou infectantes, no uso continuado de máquinas. São exemplos destas complicações as cardiopatias, as doenças vasculares periféricas, a intoxicação pelo alumínio, a imunodepressão e a neoplasia renal.

Para Talbot (2001, p.171) deve-se atentar para a avaliação do acesso vascular, que constitui fonte de problemas como infecção, sangramento e perda da perviedade. Para Fermi (2003, p. 109) constitui também um problema especial a anemia que os pacientes apresentam devido principalmente à ausência de eritropoetina.

Segundo Daugirdas (1996, p.341) “... os pacientes submetidos ao tratamento dialítico tem uma vida bastante alterada. Aqueles submetidos à hemodiálise, diálise peritoneal noturna (NIPD) ou diálise peritoneal continua assistida por cicladora (CCPD) encontram-se presos à uma máquina numa intensidade sem precedentes na história da tecnologia médica." O autor também afirma que: "...todos os pacientes de diálise consideram-se extremamente dependentes de um procedimento médico, de um hospital ou de um grupo de pessoas da área médica, além de estarem expostos a outros tipos de estresse."

A dependência do tratamento, a perda da liberdade, do emprego e da expectativa de vida são alguns dos contribuintes para o aparecimento freqüente de problemas psicológicos nesses pacientes. Fermi (2003, p. 113-114) descreve, que o paciente renal crônico geralmente apresenta depressão por perder o emprego e a capacidade de excreção renal.

De acordo com Carpenito (2002, P. 366); 
Doenges \& Moorhouse (1999, p.483-485) o paciente portador de insuficiência renal crônica sob tratamento hemodialitico apresenta muitas intercorrências como: desequilíbrio hídrico/ eletrolítico, sangramento gatrointestinal, hiperparatireoidismo, fraturas patológicas, desnutrição, anemia, sobrecarga de líquidos, hipoalbuminemia, polineuropatia, insuficiência cardíaca congestiva, edema pulmonar, acidose metabólica, derrame pleural, pericardite e tamponamento cardíaco.

A relação entre o trabalho e a saúde há tempos é fonte de preocupação de muitos, pois a saúde é sem duvida a condição fundamental para a produtividade do homem, e que muitas vezes fica prejudicada em algumas condições patológicas, como é o caso na insuficiência renal crônica. Esta preocupação segundo WISNER (2003, p.11) deve-se essencialmente à evolução da tecnologia, onde a relação com tipos antigos ou recentes de organização do trabalho cria situações não só caracterizada pelo esforço puramente físico ou mental, mas também com um componente cognitivo intenso e complexo.

O trabalho segundo Morin (2001), é um valor importante nas sociedades ocidentais contemporâneas, e exerce influência considerável sobre a motivação dos trabalhadores, assim como sobre sua satisfação e sua produtividade.

Rodrigues (1995, p.11) acrescenta e caracteriza o trabalho como algo realmente fundamental para o homem:

"A nossa relação com o trabalho parece algo ainda bastante conflitivo, sendo muitas vezes percebido como indesejado, como um fardo pesado, que acaba nos impedindo de viver. Mas freqüentemente ele é também percebido como algo que dá sentido á vida, eleva o status, define a identidade pessoal e impulsiona o crescimento humano. (...). O homem com seu trabalho não somente usufrui dos recursos da natureza, como também pode ser capaz de modificá-la, recria-la e também, infelizmente, destruíla. A ação humana apresenta-se distinta do comportamento dos outros animais porque o homem age de acordo com finalidades e planeja suas atividades. Ao modificar a natureza, ele cria a cultura, a linguagem, a historia e a si mesmo. "

Souto (2003, p. 38-39) sob este mesmo prisma apresenta a importância do trabalho no aspecto individual e social. No ponto de vista individual, o trabalho engrandece a maneira de viver do homem. No ponto de vista social, o trabalho é um dos grandes motivos para a vida coletiva e tem como principal finalidade a segurança da produção em proveito da coletividade e do Estado. Conseqüentemente com este processo surgiu a preocupação da Saúde Publica relacionadas diretamente com as conseqüências da industrialização e com demandas por melhoria das condições de vida e trabalho da população envolvida no sistema produtivo. Sob a nova sociedade capitalista, os direitos humanos á vida e á subsistência foram repensados, pois a sociedade industrial criara a necessidade de novos direitos contra riscos que anteriormente não eram considerados como significantes à vida do então chamado individuo trabalhador (MIRANDA, 1999, p.2-3).

Segundo Brasil (2002, p. 7), o termo Saúde do Trabalhador refere-se:

... a um campo do saber que visa compreender as relações entre o trabalho e o processo saúde/doença. Nesta acepção, considera a saúde e a doença como um processo dinâmico, estreitamente articulados com os modos de desenvolvimento produtivo da humanidade em determinado momento histórico. Parte do principio de que a forma de inserção dos homens, mulheres e crianças nos espaços de trabalho contribuem decisivamente para formas especificas de adoecer e morrer.

Para Souto (2003, p.37) "o trabalho é entendido como todo esforço que o homem no exercício de sua capacidade física e mental executa para atingir seus objetivos em consonância com os aspectos éticos. Todo homem tem direito ao trabalho digno, a fim de realizar-se e garantir sua subsistência assim como daqueles por quem é responsável", mas em contrapartida, o trabalhador só consegue realizar suas atividades laborais se possuir condições físicas, mentais e sociais articuladas aos fatores intrínsecos do trabalho como realização completa da jornada de trabalho, o que muitas vezes para o paciente renal crônico estas condições não são possíveis.

\section{TRAJETÓRIA METODOLÓGICA}

Pesquisa de carater descritivo. Para Tomasi \& Yamamoto (1999, p.31) "a pesquisa descritiva observa, registra, analisa e correlaciona fatos ou fenômenos sem manipulá-los". Ainda segundo a autora este tipo de pesquisa busca conhecer as diversas situações e relações que ocorrem na vida social, política, econômica e demais aspectos do 
comportamento humano individualmente , como de grupos e comunidades mais complexas.

A instituição campo de estudo foi um Centro Especializado de Hemodiálise, localizado em Curitiba, capital do estado do Paraná, que tem como finalidade promover o tratamento dialitico à pacientes portadores de Insuficiência Renal Crônica.

O quadro de funcionários da instituição é de 60 funcionários, sendo que 34 (50\% do total) pertencem à equipe de Enfermagem. O sistema de trabalho da equipe de Enfermagem está organizado com base na divisão funcional de tarefas, cabendo à coordenadora de Enfermagem a organização dos serviços administrativos gerais.

Foram entrevistados 43 pacientes portadores de Insuficiência Renal Crônica de ambos os sexos, de diferentes classes sociais e raças, submetidos à hemodiálise no campo de estudo no período da coleta.

Inicialmente foi encaminhado o projeto ao Comitê de Ética em Pesquisa para análise e aprovação. Os preceitos éticos de sigilo e anonimato dos sujeitos foram respeitados e o projeto iniciou-se após o parecer favorável à realização do estudo pelo Comitê de Ética em Pesquisa, conforme Resolução 196/96 MS.

\section{RESULTADOS E DISCUSSÕES}

A maior população estudada pertencia ao sexo masculino $(74,42 \%)$ e 11 pacientes do sexo feminino (25,58\%). Castro et.al.(2003) também obteve em seu estudo com 184 pacientes sobre a qualidade de vida dos pacientes renais crônicos em hemodiálise uma maior predominância do sexo masculino neste tratamento.

Encontramos um maior número de pacientes na faixa etária de 18 a 59 anos, correspondendo a $74 \%$ da população de estudo. Esta faixa etária abrange a faixa em que consideramos os indivíduos com maior capacidade produtiva para o mercado de trabalho bem como para outras atividades como cidadão.
Tabela 1 - Tempo de tratamento dialítico em meses dos pacientes. Curitiba, 2005.

\begin{tabular}{c|c|c}
\hline $\begin{array}{c}\text { Tempo de } \\
\text { tratamento dialítico } \\
\text { (em meses) }\end{array}$ & $\mathbf{N}^{\circ}$ de pacientes & $\%$ \\
\hline $01-40$ & 26 & 60,46 \\
$41-80$ & 10 & 23,25 \\
$81-120$ & 3 & 6,97 \\
120 ou mais & 4 & 9,32 \\
\hline TOTAL & $\mathbf{4 3}$ & $\mathbf{1 0 0}$ \\
\hline
\end{tabular}

$\mathrm{Na}$ TABELA 1, apresentamos o tempo determinado em meses que a população entrevistada, está em tratamento dialítico. A doença crônica conforme já apresentado na revisão de literatura, leva a uma dependência da hemodiálise para a sobrevida dos pacientes. Daugirdas (1996, p.341) refere que: "...pacientes submetidos à hemodiálise, diálise peritoneal noturna e diálise peritoneal contínua assistida por cicladora são pessoas que se encontram presas á uma máquina em uma intensidade sem precedentes na história da tecnologia médica". A presença de uma doença crônica segundo Castro et al (2003), está associada a uma piora da qualidade de vida de uma população, o que pode justificar a redução do número de pacientes em hemodiálise de acordo com o aumento da faixa etária da população deste estudo. Também segundo a autora o que contribui substancialmente para o aumento da sobrevida dos pacientes renais crônicos, são os avanços na área de diálise.

Tabela 2 - Freqüência dos diagnósticos clínicos segundo o número de pacientes. Curitiba, 2005.

\begin{tabular}{l|c|c}
\hline $\begin{array}{l}\text { Diagnósticos } \\
\text { Clínicos }\end{array}$ & $\mathbf{N}^{\circ}$ de pacientes & $\%$ \\
\hline $\begin{array}{l}\text { Glomerulonefrites } \\
\text { Nefroesclerose }\end{array}$ & 20 & 46,51 \\
$\begin{array}{l}\text { hipertensiva } \\
\text { Outras patologias } \\
\text { como nefropatias } \\
\text { por refluxo, }\end{array}$ & 7 & 23,25 \\
$\begin{array}{l}\text { uropatias e } \\
\begin{array}{l}\text { Miefomeffites } \\
\text { Diabética }\end{array}\end{array}$ & 6 & 16,27 \\
\hline TOTAL & $\mathbf{4 3}$ & $\mathbf{1 0 0}$ \\
\hline
\end{tabular}


A TABELA 2 mostra o número de pacientes segundo as patologias que desencadearam a insuficiência renal crônica. Os diagnósticos clínicos que compõem esta tabela foram retirados dos prontuários dos pacientes pois muitos pacientes não souberam referir o diagnóstico quando entrevistados.

Ao contrário, dos escritos de Guyton \& Hall (1996, p. 379), a maior causa de insuficiência renal crônica é a doença renal crônica hipertensiva evoluindo para nefroesclerose hipertensiva. A população estudada apresentou uma maior incidência de diagnóstico por glomerulonefrites. Importante ressaltar para a análise destes dados que as glomerulonefrites são classificadas em primárias e secundárias, nas primárias predomina o mecanismo imunológico, humoral ou celular, há produção de anticorpos contra os componentes do glomérulo ou contra antígenos externos ou internos com a formação de imunocomplexos circulantes que serão depositados nos rins, causando dano glomerular. Nas secundárias o mecanismo dependerá da doença sistêmica (diabete, lúpus, neoplasias, hepatopatias, etc). Em geral há alterações da permeabilidade e seletividade do capilar, o que eleva a passagem anormal de elementos do sangue na urina, há redução na superfície de filtração glomerular e alterações na regulação da excreção de água e sódio causando edema e hipertensão (BARROS, 1999).

Portanto pode se associar a maior ocorrência de glomerulonefrites a ocorrência simultânea de outras patologias como o diabetes, o que vem de encontro com outros autores como Riella (1996), que associam a insuficiência renal como uma disfunção fisiológica correlacionada com outras patologias como a hipertensão arterial sistêmica. Estas patologias com o passar dos anos desencadeiam a insuficiência renal crônica.
Tabela 3 - Número de pacientes em hemodiálise

\begin{tabular}{|c|c|c|}
\hline Escolaridade & $\mathrm{N}^{\circ}$ de pacientes & $\%$ \\
\hline 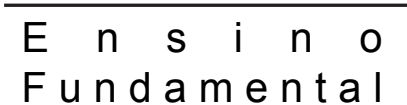 & 21 & 48,83 \\
\hline $\begin{array}{l}\text { Erasinmpletm damental } \\
\text { Completo }\end{array}$ & 8 & 18,60 \\
\hline Ensino Médio & 2 & 4,65 \\
\hline Incompleto & & \\
\hline Ensino Médio & 8 & 18,60 \\
\hline $\begin{array}{l}\text { Ensino Superior } \\
\text { Incompleto }\end{array}$ & 2 & 4,65 \\
\hline $\begin{array}{l}\text { Ensino Superior } \\
\text { Completo }\end{array}$ & 2 & 4,65 \\
\hline TOTAL & 43 & 100 \\
\hline
\end{tabular}

Segundo os dados encontrados, aproximadamente $67 \%$ da amostra obteve apenas acesso ao ensino fundamental. Encontramos nesta amostra significativa que estes pacientes apresentam uma maior dificuldade para compreender toda a extensão do procedimento hemodialítico. Nesta população encontramos também grande dificuldade no seguimento das orientações preconizadas pela equipe de saúde, o que pode comprometer ainda mais o seu estado de saúde. Uma pequena população de $23 \%$, consegue assimilar alguns aspectos da doença e consegue melhor resposta no tratamento, os pacientes que possuem algum conhecimento intelectual, isto vem de encontro com Castro et al (2003), que afirma uma melhor resposta à hemodiálise os pacientes com algum nível cultural. Este fato confirma a importância da reflexão dos profissionais envolvidos neste tratamento, quanto à análise das condições socioeconômicas anteriores ao tratamento, apresentadas por estes pacientes . 
Gráfico 1 - Presença de atividade laboral entre os pacientes estudados. Curitiba, 2005.

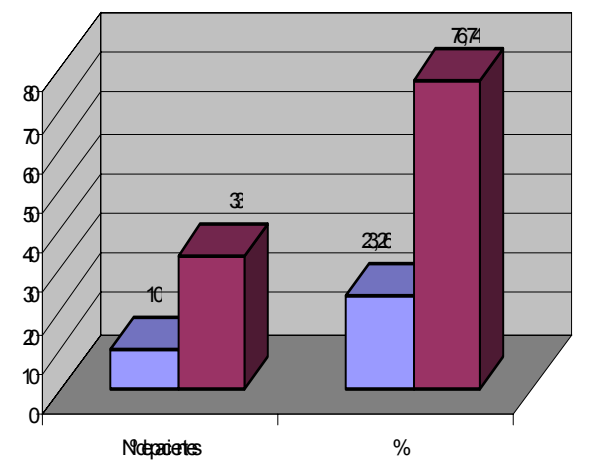

Q Pessidivicatdand

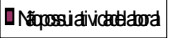

O GRÁFICO 1 mostra a freqüência percentual da realização da atividade laboral pela população da amostra. A maior freqüência percentual da amostra $(76,74 \%)$ não possui atividade laboral, ou seja, segundo estes dados a população submetida à hemodiálise encontra-se inativa sob o ponto de vista econômico, não possuindo fonte de renda, o que pode comprometer muito seu tratamento.

Como citado anteriormente neste estudo, a atividade laboral tem um papel fundamental na vida do homem. Sendo assim, quando o ser humano por alguns fatores inerentes à sua vontade, por alterações fisiológicas, quando este indivíduo passa a ter sua vida bloqueada para uma condição incapacitante, surgem as perturbações da identidade e da auto-estima. Como já abordado por Daugirdas (1996, p.341), Fermi (2003, 113-14), são as principais causas de transtornos emocionais a perda do emprego e da capacidade de micção.

Foram feitos alguns questionamentos quanto a renda e sua adequação as necessidades do paciente. Muitos não apresentavam renda financeira e a maioria tem como renda familiar um salário mínimo que recebe por aposentadoria ou auxilio-doença. Perante estes dados pode-se concluir que para esta população a hemodiálise traz mudanças não só para o estilo de vida do individuo em tratamento mas como também para a adequação da nova renda que muitas vezes é bem menor que a anterior ao período da instalação da doença crônica para ele e para a sua família.Também foram feitos questionamentos em relação à ajuda financeira recebida por outros, encontramos que apenas 5 indivíduos desta amostra referiram receber alguma ajuda de familiares e amigos.

Tabela 4 - Freqüência dos motivos relatados pelos pacientes em estudo para a não realização da atividade laboral. Curitiba, 2005.

\begin{tabular}{l|c|c}
\hline $\begin{array}{l}\text { Motivos } \\
\text { relatados }\end{array}$ & $\begin{array}{l}\mathbf{N}^{\circ} \text { de } \\
\text { pacientes }\end{array}$ & $\begin{array}{c}\% \text { da } \\
\text { amostra* }\end{array}$ \\
\hline $\begin{array}{l}\text { Não apresenta } \\
\text { condições físicas } \\
\text { para executar a } \\
\text { atividade laboral } \\
\text { exigida }\end{array}$ & 18 & 54,55 \\
$\begin{array}{l}\text { Não consegue } \\
\text { cumprir jornada de } \\
\text { trabalho exigida } \\
\text { Já estava } \\
\text { aposentado ou } \\
\text { desempregado } \\
\text { antes de iniciar o } \\
\text { tratamento }\end{array}$ & 7 & 21,20 \\
\hline TOTAL & 8 & 24,25 \\
\hline
\end{tabular}

NOTA: *Nesta tabela foi considerada como população de amostra somente os pacientes que não realizam atividade laboral, totalizando 33 pacientes, dos 43 estudados.

Uma porcentagem significativa $(54,55 \%)$ da população não trabalha porque não apresenta condições físicas para realizar a atividade laboral. Alguns pacientes relataram que seus serviços exigiam muita atividade braçal, mas com a presença da fístula arteriovenosa a atividade laboral se tornara impossível de realizar.

Outros relataram a dificuldade de manter o emprego, devido ao tempo dispendido na hemodiálise, muitos pacientes utilizam pelo menos 6 horas do dia ao tratamento, pois após as 4 horas de hemodiálise este paciente leva aproximadamente mais 2 horas para a recuperação dos sintomas imediatos após o tratamento.

Uma pequena população de estudo $(24,25 \%)$, referiu já não realizar nenhuma atividade laboral no inicio do tratamento e após ter iniciado o procedimento dialítico, ficou ainda mais difícil esta inserção no mercado de trabalho.

Encontramos também que a maioria dos pacientes $(56 \%)$ após o tratamento hemodialítico 
apresenta sintomas clínicos como presença de mal-estar, quedas da pressão e câimbras. Constatamos que estas intercorrências fisiológicas podem comprometer as atividades laborais, pois foi confirmado que estas intercorrências relatadas pelos pacientes impossibilitam a realização da atividade laboral, pois muitos relataram cansaço necessitando de repouso após as sessões de hemodiálise.

\section{CONSIDERAÇÕES FINAIS}

A insuficiência renal crônica é uma doença que pode comprometer a atividade laboral do paciente e que reduz assim como outras patologias crônicas a qualidade de vida do homem. A pessoa portadora de insuficiência renal crônica e submetida à hemodiálise passa por uma série de transformações nos seus hábitos de vida, o que a obriga a conviver com um procedimento doloroso e com grandes restrições impostas pela doença.

Neste estudo constatamos o comprometimento da capacidade funcional principalmente dos indivíduos considerados economicamente ativos na sociedade. $O$ objetivo deste estudo foi alcançado, pois ocorrem mudanças significativas na vida do paciente quando inicia a hemodiálise comprometendo a qualidade de vida, e principalmente dificultando sua atividade laboral.

ABSTRACT: The present study looked for the understanding of the changes that the hemodialisis brought to the accomplishment of activities work for the renal chronic patient of a Center of Hemodialisis in Curitiba. The study was descriptive quantitative. The studied population was of 43 patients that did hemodialisis sessions. In the obtainable results there were evidenced the difficulties that these patient ones present to maintain the activity laborer after beginning the treatment. Most of the population is retired by disability, it receives sick pay or it waits for retirement. The patient's interviewees that possess activity laborer refer to have difficulties to accomplish them due to the impossibility of maintaining a work day adapted to their condition. They also present weakness after the hemodialisis sessions. It was characterized that most of the patients has difficulties to maintain financially, once they can't develop the activities work.

KEY WORDS: Hemodialisis, Chronic renal inadequacy; Occupational health.

\section{REFERÊNCIAS}

BARROS, E. et al. Nefrologia: rotinas, diagnóstico e tratamento. 2 ed. Porto Alegre. Artes Médicas Sul, 1999.

BRASIL.Ministério da Saúde.Caderno de Atenção Básica-Saúde do Trabalhador. № 5 . Brasília, 2002.

CASTRO, M. et. al Qualidade de vida de pacientes com Insuficiência Renal Crônica em Hemodiálise avaliada através do instrumento genérico SF-36. Revista da Associação Médica do Brasil. Vol.49 $-n^{\circ}$ 3. 2003.

CARPENITO LJ. Diagnóstico de enfermagem: aplicação à prática clínica. 6. ed. Porto Alegre: Artmed, 2000

DAUGIRDAS, J. T.; ING, T. S. Manual de Diálise . 2 ed. Rio de Janeiro. Medsi, 1996.

DOENGES, M.;MOORHOUSE, M.F. Diagnóstico e Intervenção em Enfermagem. 5 ed. São Paulo. Artmed, 1999.

FERMI, M. R. V. Manual de Diálise para Enfermagem. São Paulo. Medsi, 2003

GUYTON, A. C.; HALL, J. E. Tratado de Fisiologia Médica. Rio de Janeiro. Guanabara- Koogan, 1996.

MIRANDA, C. R. Introdução á Saúde do Trabalhador. São Paulo. Atheneu, 1999.

MORIN, E. M. Os sentidos do Trabalho. Revista de Administração de Empresas - FGV. Vol 41n³. Julho- Setembro 2001.

RIELLA, C. M. Princípios de Nefrologia e Distúrbios Hidroeletroliticos. 3 ed. Rio de Janeiro. Guanabara - Koogan, 1996. 
SOUTO, D. F. Saúde no Trabalho: uma revolução em andamento. Rio de Janeiro. Senac/ Sesi Nacional, 2003.

TALBOT, L.A.; MARQUARDT, M.M. Enfermagem Prática: Avaliação em Cuidados Críticos. 3 ed. Rio de Janeiro. Reichmam \& Affonso, 2001.

TOMASI, N.G.S.; YAMAMOTO, R.M. Metodologia da Pesquisa em Saúde: Fundamentos Essenciais. Curitiba. As autoras, 1999.

ZATZ, R. Fisiopatologia Renal. 2 ed. São Paulo. Atheneu, 2002.

WISNER A. A inteligência no trabalho: textos selecionados de ergonomia. São Paulo: Fundacentro, 1994. 